\title{
Situation-Aware Adaptive Processing (SAAP) of Data Streams
}

\author{
Pari Delir Haghighi, Mohamed Medhat Gaber, Shonali Krishnaswamy and \\ Arkady Zaslavsky
}

\begin{abstract}
The growth and proliferation of technologies in the field of sensor networking and mobile computing have led to the emergence of diverse applications that process and analyze sensory data on mobile devices such as a smart phone. However, the real power to make a significant impact on the area of developing these applications rests not merely on deploying the technologies but on the ability to perform real-time, intelligent analysis of the data streams that are generated by the various sensors. In this chapter, we present a novel approach for SituationAware Adaptive Processing (SAAP) of data streams for pervasive computing environments. This approach uses fuzzy logic principles for modeling and reasoning about uncertain situations and performs gradual adaptation of parameters of data stream mining algorithms in real-time according to availability of resources and the occurring situations.
\end{abstract}

\section{Introduction}

There is a range of emerging applications that use mobile devices for data analysis and processing of data streams in ubiquitous computing environments. Examples of such applications include mobile fieldworkers (e.g. healthcare professionals, firefighters, police, etc.), intrusion detection and Intelligent Transportation Systems. A very significant challenge for these applications is to process and analyze the vast amounts of data streams that are typically generated at very high rates with mobile devices such as a PDA in real-time and in a smart and cost-efficient way. Lightweight and one-pass data stream mining algorithms such as LWC and LWCLass [1-2] that are developed for Ubiquitous Data Stream Mining (UDM) [3] are able to perform real-time analysis on-board small/mobile devices while considering available resources such as battery charge and available memory. However, to perform smart and intelligent analysis of data on mobile devices, it is imperative for adaptation strategies to go beyond mere computational resources and factor in contextual/situational information.

Context can be any information related to a network, environment, application, process, user or device. Here we consider context as the information used for representing real-world situations [4] in pervasive computing environments. Con- 
textual information collected from every single sensor or data source represents a partial view of the real-world. Aggregation of data from multiple sensors and sources provides a wider and more general view of surrounding environment and situations of interest [5]. For example, in a smart room scenario, rather than monitoring sensed context from light, noise and motion sensors individually, this information can be used to reason about situations such as 'meeting', 'presentation' or 'study' which provides a better understanding of the environment. As a metalevel concept over context, we define the notion of a situation that is inferred from contextual information [5]. Situation-awareness provides applications with a more abstract view of their environment rather than focusing on individual pieces of context.

Reviewing recent works in mobile data stream mining reveals that most of these projects [1-4] have limited levels of adaptations or mainly focusing on the battery or memory usage. A general approach for smart and cost-efficient analysis of data that is under-pinned using situation-aware adaptation has not been introduced in the current state-of-the-art and is still an open issue.

To incorporate situation-awareness into ubiquitous data stream mining, we have introduced a general approach called Situation-Aware Adaptive Processing (SAAP) of data streams. This chapter is devoted to introduce the architecture of SAAP and the techniques we apply to achieve situation-awareness and adaptation of data stream mining algorithms. The main contributions of the SAAP architecture are as follows:

- Modeling and reasoning about situations are achieved using a Fuzzy Situation Inference (FSI) model that is based on the Context Spaces (CS) model [5], a general and formal approach for reasoning about context under uncertainty. The FSI technique incorporates the CS model's underlying theoretical basis for supporting context-aware and pervasive computing environments while using fuzzy logic to model and reason about imperfect context and vague situations;

- Parameterized adaptation of data stream mining algorithms is performed in real-time and according to the occurring situations and available resources. This approach maximizes the benefits of adaptation and improves data stream mining operations in an intelligent and cost-efficient manner. Situation-aware adaptation leverages the full potential of UDM and can enable, if not guarantee, the continuity and consistency of the running application.

This chapter is organized as follows. Section 2 describes the SAAP architecture. Section 3 discusses the Fuzzy Situation Inference Engine (FSIE) that enables situation-awareness using fuzzy logic principles. Section 4 discusses parameterized adaptation of data stream mining algorithms factoring in situations and resources. Section 5 describes implementation and evaluation of the architecture. Finally section 6 provides the summary of the chapter. 


\section{Situation-Aware Adaptive Processing (SAAP) of Data Streams}

The architecture for Situation-Aware Adaptive Processing (SAAP) of data streams consists of two main components of Fuzzy Situation Inference (FSI) and Adaptation Engine (AE) as shown in Fig. 1. FSI enables situation-awareness by combining fuzzy logic principles with the Context Spaces (CS) model. The second component, $\mathrm{AE}$, is responsible for gradual and parameterized adaptation of data stream mining algorithms in real-time according to the occurring situations and available resources.

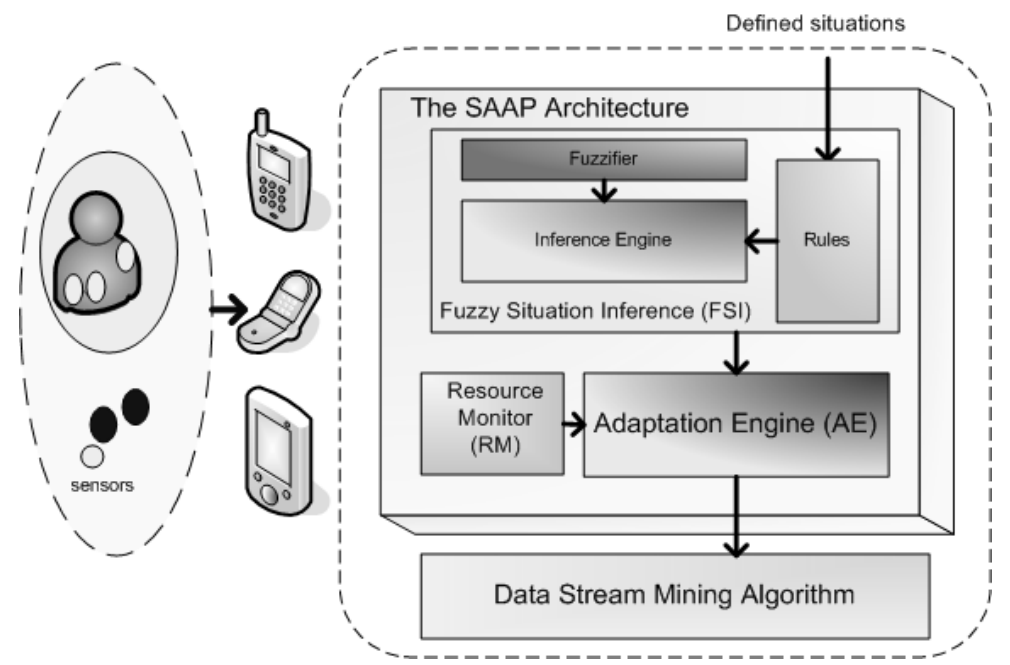

Fig. 1. The architecture of for Situation-Aware Adaptive Processing (SAAP) of data streams.

Resource Monitor (RM) is a software component that continuously monitors available resources such as available memory and battery usage and reports their availability to the adaptation engine. The SAAP layer is built on the top of the data stream mining algorithms running on mobile devices and provides them with situation-aware adaptation.

\section{Fuzzy Situation Inference (FSI)}

The Fuzzy Situation Inference (FSI) is a situation modeling and reasoning approach that integrates fuzzy logic into the Context Spaces model [5]. The strengths of fuzzy logic for modeling and reasoning of imperfect context and vague situations are combined with the CS model's underlying theoretical basis for supporting context-aware pervasive computing scenarios. 
One of the main challenges in enabling situation-awareness in pervasive applications is managing uncertainty. Uncertainty can be related to context imperfection such as sensors' inaccuracy, missing information or imperfect observations [6]. However, there is another dimension of uncertainty that is inherent in human concepts and every day situations. In real-world, situations evolve and change into other situations (e.g. 'walking' changes to 'running'). Changes that occur between situations of 'walking' and 'running' are also good indicators of situations that may emerge - albeit with some vagueness and uncertainty. These uncertain situations can be of high importance to certain applications such as a health monitoring application that needs to monitor details of changes in a patient's health situation. The CS model mainly deals with uncertainty associated with sensors' inaccuracy. In order to model real-world situations and reflect this aspect of uncertainty in the situation reasoning results we combine the CS modeling and reasoning techniques with fuzzy logic principles. Next subsection introduces the Context Spaces (CS) model and its main concepts.

\subsection{The Context Spaces (CS) Model}

The CS model represents contextual information as geometrical objects in multidimensional space called situations [5]. The basic concepts of the CS model are the context attribute, application space, context state and situation space. A context attribute describes any data used in the situation reasoning. The term application space defines the universe of discourse and context state refers to a collection of context values in CS. The concept of a situation space is characterized by a set of regions. Each region is a set of acceptable values of a context attribute that satisfies a predicate. A region is a crisp or conventional set of context attribute values such that any element is its member or not. For example, in a situation, a region of temperature can be the values between 15 and 25 that satisfy two predicates of ' $>15$ ' and ' $<25$ '.

In addition to basic concepts and techniques for situation modeling and reasoning, the CS model provides heuristics developed specifically for addressing context-awareness under uncertainty. These heuristics are integrated into reasoning techniques that are utility-based data fusion algorithms and compute the confidence level in the occurrence of a situation [5]. Main heuristics of the CS model are as follows:

- Individual significance (i.e. weight) and contribution of context attributes in the situation space;

- Inaccuracies of sensory originated information;

- Characteristics of context attributes and their effect on reasoning;

- Partial and complete containment of context-attributes' values in the situation space. 
Each of the above-mentioned heuristics is used in a reasoning technique of CS to compute the confidence level in the occurrence of a situation. To enable situation-awareness in pervasive applications, it is imperative to address the issue of uncertainty. The CS deals with uncertainty mainly associated with sensors' inaccuracies. Yet there is another aspect of uncertainty in human concepts and realworld situations that needs to be represented in a context model and reflected in the results of situation reasoning. Fuzzy logic has the benefit of representing this level of uncertainty using membership degree of values. The next section describes situation modeling in FSI.

\subsection{Situation Modeling}

Definitions of 'application space' and 'context state' are applied similarly to the FSI model but the 'situation space' is differently defined. In FSI, a situation is defined by a set of fuzzy sets that are expressed as a FSI rule. In a fuzzy set, unlike a region, membership of an item is gradual and is represented by a membership degree between 0 and 1 [7]. Using fuzzy sets allows a context value to be represented both qualitatively as fuzzy terms and quantitatively using membership functions. The FSI consists of three subcomponents of fuzzifier, rules and inference engine. The next subsections discuss these parts in more detail.

\subsubsection{Fuzzifier}

In FSI, crisp inputs are context attribute values such as temperature degree or light level that are obtainable by the application. Fuzzifier, as a software component, uses membership functions to map crisp inputs (i.e. context attribute values) into fuzzy sets. Prior to fuzzification, we need to define linguistic variables and their terms and then select appropriate membership functions for mapping crisp inputs into fuzzy sets.

- Defining Linguistic variables: Zadeh [8], the founder of the field of fuzzy logic, defines linguistic variables as:

"variables whose values are not numbers but words or sentences in a natural or artificial language."

In the FSI model, the term linguistic variable is used to express a 'context attribute'. Unlike context attributes, values that linguistic variables take are not numeric and are called terms (also known as fuzzy variables) [9]. Each term of a linguistic variable represents a fuzzy set that takes a pair of numeric values (i.e. a value and its membership degree). For a situation of 'hyperthermia', linguistic variables can be 'heart-rate', 'room temperature' and 'age'. 
- Breaking linguistic variables into terms: after defining linguistic variables, we need to break them into terms or fuzzy variables. Each term of a linguistic variable is characterized by a fuzzy set defined on U. For example, the terms of the linguistic variable 'heart rate' could be \{'slow', 'normal' and 'fast' $\}$.

- Selecting membership functions: a fuzzy set is characterized by a membership function that assigns to each object a grade of membership [7, 9]. Let A be a fuzzy set defined on a universe of $U$ and $x$ be an element of the universe. A membership function denoted as $\mu_{A}(x)$ maps $x$ to a membership grade between 0 and 1 [7, 9]. If 'heart rate' is $87 \mathrm{bpm}$, then we could have $\mu_{\text {normal }}(87)=0.3$ and $\mu_{\text {fast }}(87)=0.8$.

There are many different varieties of membership functions such as trapezoidal, Gaussian and Bell. Selecting a membership function is subjective [7] and is based on the application requirements and expected membership degree for the values. We consider the trapezoidal membership function the most fitting function for characterizing our fuzzy sets with the following format:

$$
\operatorname{trapmf}(x ; a, b, c, d)=\max \left(\min \left(\frac{x-a}{b-a}, 1, \frac{d-x}{d-c}\right), 0\right)
$$

The parameters passed into the trapezoidal function determine the membership degree of elements. The greater the membership degree of elements, the greater confidence and evidence that the situation is occurring. As shown in Fig. 2, the parameters ' $a$ ' and ' $d$ ' represent the 'feet' and ' $b$ ' and 'c' locate the 'shoulders' of the trapezoid. The membership degree of values across the 'shoulders' is 1 and the membership degree of values towards the 'feet' reduces from 1 to 0 .

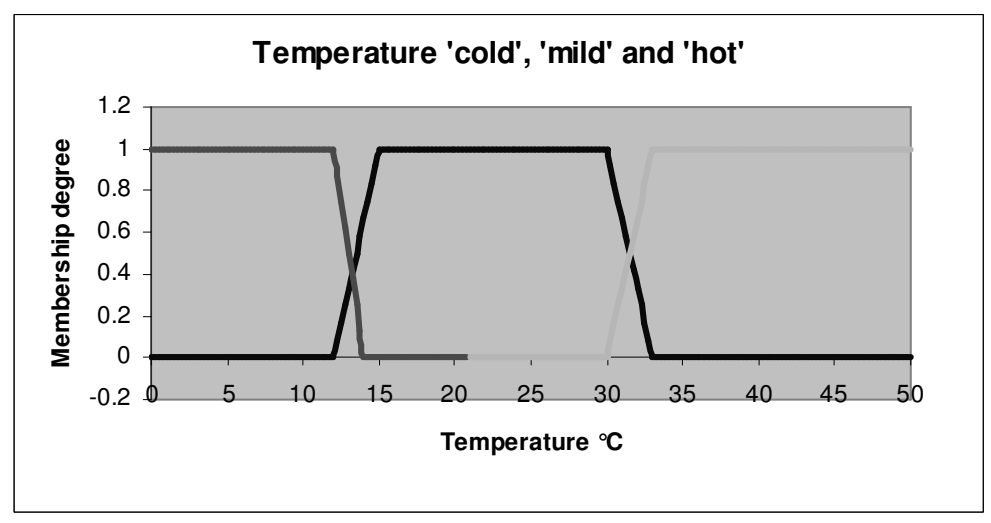

Fig. 2. An example of a trapezoidal membership function. 


\subsubsection{Rules}

In fuzzy logic, rules are "if-then" statements whose return values are not restricted to "true" or "false" and can be a value between 0 and 1. In FSI, we use fuzzy rules to represent situations of interest. Each FSI rule consists of multiple conditions/antecedents joined with the AND operator but a condition can itself be a disjunction of conditions [10]. An example of a FSI rule for a health-related situation can be expressed as follows:

Rule1: if RT is 'hot' and HR is 'fast' and (age is 'middle-aged' or 'old) then situation is 'heat-stroke'

In many cases, there are some fuzzy variables that are more important than others in describing a situation. For example, low blood pressure is a strong indication of 'hypotension' in a person while heart rate may not be equally important. To model the importance of fuzzy variables and conditions, we assign a predefined weight $w$ to each condition with a value ranging between 0 and 1 . The sum of weights is 1 per rule. A weight represents the importance of its assigned condition relative to other conditions in defining a situation.

In pervasive environments, an application's knowledge about its environment is partially based on the information that it collects from sensors. However, due to sensor faults or inaccuracies this information can be incomplete or unavailable. This requires the situation modeling and reasoning approaches to provide more flexibility such that if one of the existing sensors fails or a new sensor is added, the system still performs reasoning based on the available information. Representing a situation with different types or cardinality of context provides an application with more flexibility to reason about situations. The FSI model supports definition of a situation with multiple rules. Each rule can consist of dependent or overlapping conditions to represent a certain situation as follows.

Rule 1: If $A$ and $B$ and $C$ then situation is $Z$

Rule 2: If $A$ and $C$ and $D$ then situation is $Z$

Rule 3: If $E$ and $F$ then situation is $Z$

Rule 4: If $E$ and $F$ and $G$ then situation is $Z$

where A, B, C, D, E, F, G are conditions and $\mathrm{Z}$ represents a situation. Each rule represents situation $\mathrm{Z}$ with different combinations of conditions. The results of evaluation of all the rules for a situation are aggregated using maximum function to produce a single result per situation.

To improve cost-efficiency aspect of the evaluation process, multiple rules are rearranged in an ascending order. The order is based on the weights of conditions because weights are the main indicators of importance of each condition in a rule. For example, if rule 1, 2 and 3 consists of three conditions with weights of $(0.36$, $0.32,0.32),(0.5,0.4,0.1)$ and $(0.4,0.4,0.2)$ respectively, these rules are rearranged as rule 2,3 and 1 . Table 1 illustrates an example of representing 'heatstroke' with multiple rules rearranged in an ascending order. The variables are RT, 
$\mathrm{HR}$, age and RR (respiratory rate) with the values of $36^{\circ} \mathrm{C}, 89 \mathrm{bpm}, 63$ and 26 brpm.

Table 1. An example of a situation defined by multiple rules

RULES FOR 'HEATSTROKE'

Rule1: if RT is hot and HR is fast and (age is middle-aged or old) then situation is hypothermia $(1 * 0.5)+(0.9 * 0.4)+\max ((0.4 * 0.1),(0.6 * 0.1))=0.92$

Rule2: if RT is hot and RR is fast and (age is middle-aged or old) then situation is hypothermia $(1 * 0.5)+(0.75 * 0.4)+\max ((0.4 * 0.1),(0.6 * 0.1))=0.86$

Rule3: if RT is hot and HR is fast and RR is fast then situation is hypothermia

$(1 * 0.36)+(0.9 * 0.32)+(0.75 * 0.32)=0.888$

Aggregation result

$\max =0.92$

Table 1 shows aggregation of rules when all the information about HR, RR, RT and age are obtainable. However, if one of the sensors, for example heart rate sensor, fails (and even if heart rate is considered as a symmetric context attribute), the system is still able to reason about the situation and the result of aggregation would be 0.86 . If we had used only one rule (i.e. rule 1 ) for representing 'heatstroke' and the sensor providing heart rate data failed, the result of inference would have been reduced to the confidence value of 0.56 that indicates a less accurate value compared to 0.86 .

\subsection{Situation Reasoning}

To reason about a situation, rules need to be evaluated to produce a single output that determines the membership degree of the consequent. The conditions joined with the OR operator are evaluated using the maximum function [7]. To evaluate the conditions joined with the AND operator, FSI provides four reasoning techniques. These techniques integrate fuzzy logic principles into the reasoning methods of CS to provide another aspect of uncertainty (i.e. uncertainty of situations and delta changes of context) in the computation of confidence value for the occurrence of a situation. The degree of confidence is a measure reflecting how many and to what extent indicative events are supportive of the specific situation.

The CS model has based its reasoning techniques on four heuristics that deal with uncertainty in pervasive computing environments. The following subsections discuss these heuristics and their corresponding reasoning methods in CS and FSI.

\subsubsection{Weights and Contribution Level}

The first heuristic of CS deals with the weights of context attributes and the level of confidence of attributes' values. Weights are values between 0 and 1 that are 
assigned to context attributes and represent relative importance of each context attribute for inferring a situation. Level of confidence is assigned to each element and reflects how that element relates to the modeled situation. This heuristic is integrated into the first reasoning technique of CS that computes the confidence level (i.e. a value between 0 and 1 ) in the occurrence of a situation as follows.

$$
\text { Confidence }=\sum_{i=1}^{n} w_{i} c_{i}
$$

where $w_{i}$ and $c_{i}$ denote the weight and contribution level of context attributes of a situation respectively. The contribution function that assigns the confidence values is proposed at a conceptual level and its implementation is later introduced in the second reasoning method based on sensors' inaccuracy.

In FSI, the concept of weights is associated with the conditions of a rule but the concept of a contribution level is implemented in a different way. The FSI equivalent to the equation (2) is a rule evaluation method that computes a level of confidence using membership functions and presented as follows.

$$
\text { Confidence }=\sum_{i=1}^{n} w_{i} \mu\left(x_{i}\right)
$$

where $\mu\left(x_{i}\right)$ denotes the membership degree of the element $x_{i}$ and $w_{i}$ represents a weight assigned to a condition. The result of $w_{i} \mu\left(x_{i}\right)$ represents a weighted membership degree of $x_{i}$ and $\mathrm{n}$ represents the number of conditions in a rule $(1 \leq \mathrm{i} \leq \mathrm{n})$.

\subsubsection{Sensors' Inaccuracy}

To provide automatic computation of the contribution level at run-time, the second reasoning technique of CS incorporates the heuristic of sensors' inaccuracy. The impact of sensor inaccuracies and unreliability is used as a determining factor to compute the contribution level in the following reasoning method:

$$
\text { Confidence }=\sum_{i=1}^{n} w_{i} \cdot \operatorname{Pr}\left(\hat{a}_{i}^{t} \in A_{i}\right) \cdot
$$

where $\operatorname{Pr}\left(\hat{a}_{i}^{t} \in A_{i}\right)$ presents the confidence level of a context attribute value by computing the probability of a context attribute correct value $\hat{a}_{i}^{t}$ being contained in the region $A_{i}$. To compute the probability value based on the reliability of a 
sensor, the reliability of reading (e.g. 95\%) is used to represent the probability value (i.e. $\operatorname{Pr}\left(\hat{a}_{i}^{t} \in A_{i}\right)=0.95$ ).

Second option to compute the probability value is to integrate the sensors' inaccuracy of reading rather than the reliability of reading. Using this option, the probability value is calculated in the following format:

$$
\operatorname{Pr}\left(e_{j} \leq a_{i}^{t}-\min \left(A_{i}^{j}\right)\right)-\operatorname{Pr}\left(e_{j} \leq a_{i}^{t}-\max \left(A_{i}^{j}\right)\right)
$$

where $a_{i}^{t}$ denotes the sensed value of the context attribute, $e_{j}$ denotes the sensor reading error (i.e. $\left.a_{i}^{t}-\hat{a}_{i}^{t}\right)$ and $\min \left(A_{i}^{j}\right)$ and $\max \left(A_{i}^{j}\right)$ represent minimum and maximum values of the region.

The CS equation (4) deals with uncertainty factoring in inaccuracies of sensors however this equation does not reflect delta changes of values in the equation and is not adequate to reason about vague situations. The FSI equivalent to the CS equation (4) not only incorporates the contribution level associated with sensors' inaccuracy but includes the membership of the values as another factor affecting the contribution level. In FSI, we first calculate the correct value based on the reliability or error rate and then pass it to the membership function as follows.

$$
\text { Confidence }=\sum_{i=1}^{n} w_{i} \mu\left(f\left(x_{i}, e_{i}\right)\right)
$$

where $w_{i}$ represents a weight assigned to a condition and $\mu\left(f\left(x_{i}, e_{i}\right)\right)$ denotes the membership degree of the element $x_{i}$. The function $f$ calculates the correct value of the context based on the inaccuracy value $e_{i}$. If $e_{i}$ is a reliability rate, the sensed value is multiplied by it and if it is an error rate (i.e. \pm ) it is added to the sensed value.

\subsubsection{Symmetric and Asymmetric Attributes}

The third reasoning technique of CS introduces the concepts of symmetric context attribute $C A_{S}$ and asymmetric context attribute $C A_{A}$. A symmetric context attribute increases the confidence in inferring a situation if its value is within the corresponding region and decreases the confidence if it is outside that region (e.g. reasoning about the 'hypertension' situation based on 'blood pressure'). An asymmetric context attribute increases the confidence in inferring a situation if its value is within the corresponding region but would not decrease the confidence if it is outside that region (e.g. reasoning about the 'heat stroke' situation based on 'age'). Whenever an asymmetric attribute is not contained within its region, the 
redistribution method assigns 0 to the weight of the attribute and recalculates the relative weights for the remaining attributes as follows.

$$
\hat{w}_{i}=w_{i} / \sum_{i=1}^{n} w_{i}
$$

The above-mentioned characteristic of context attributes is integrated into the following reasoning technique of CS:

$$
\text { Confidence }=\sum_{i=1}^{n} \hat{w}_{i} \cdot \operatorname{Pr}\left(\hat{a}_{i}^{t} \in A_{i}\right)
$$

where $a_{i} \in C A_{S} \cup C A_{A}$ and $\hat{w}$ denotes the recalculated weight.

The concept of symmetric and asymmetric context attributes and its corresponding reasoning techniques is applied to FSI as follows.

$$
\text { Confidence }=\sum_{i=1}^{n} \hat{w}_{i} \mu\left(f\left(x_{i}, e_{i}\right)\right)
$$

where $x_{i} \in F S$ and $F S \in L V_{S} \cup L V_{A}$. Since values that linguistic variables take are not numeric (i.e. these values are called terms that represent fuzzy sets), the concept of symmetric and asymmetric concepts are applied to the values of fuzzy sets associated with linguistic variables.

\subsubsection{Partial and Symmetric and Asymmetric Attributes}

The fourth reasoning technique of CS is based on the notion that there is a tradeoff between complete containment of all symmetric context attributes (i.e. when all values of symmetric attributes are contained in their corresponding regions) and their individual contribution using the third reasoning technique (i.e. when some of values of attributes are contained). This heuristic does not apply to asymmetric attributes because they do not decrease the confidence for the occurrence of a situation. The reasoning technique based on partial and complete containment is as follows.

$$
\text { Confidence }=q_{1} \sum_{i=1}^{n} \hat{w}_{i} \cdot \operatorname{Pr}\left(\hat{a}_{i}^{t} \in A_{i}\right)+q_{2} \prod_{k=1}^{m} \operatorname{Pr}\left(\hat{a}_{k}^{t} \in A_{k}\right)
$$

where $a_{i} \in C A_{S} \cup C A_{A}, a_{k} \in C A_{S}$ and $q_{1}+q_{2}=1 . q_{1}$ and $q_{2}$ denote the utility weights used to represent each aspect of containment with a dimension. Apply- 
ing these dimensions addresses the trade-off between complete and partial containment and combines them towards inferring the occurrence of a situation. The utility weights of two dimensions determine which aspect of containment is more important (i.e. complete or partial).

The concept of partial and complete containment and its reasoning technique are applied to FSI and similar to the third reasoning method, FSI maps values of symmetric context attributes into the values of fuzzy sets corresponding to symmetric linguistic variables as follows.

$$
\text { Confidence }=q_{1} \sum_{i=1}^{n} \hat{w}_{i} \cdot \mu\left(f\left(x_{i}, e_{i}\right)+q_{2} \prod_{k=1}^{m} \mu\left(f\left(x_{k}, e_{k}\right)\right)\right.
$$

where $q_{1}+q_{2}=1, \quad x_{i} \in F S \quad$ and $F S \in L V_{S} \cup L V_{A}, x_{k} \in F S \quad$ and $F S \in L V_{S}$.

Results of computation of the above-mentioned reasoning techniques suggest the degree of confidence in the occurrence of a situation. In FSI, if the output of a rule evaluation for the 'hypertension' situation yields a degree of 0.885 , we can suggest that the level of confidence in the occurrence of 'hypertension' is 0.885 . This value can be compared to a confidence threshold $\varepsilon$ between 0 and 1 (i.e. predefined by the application's designers) to determine whether a situation is occurring.

$$
\mu_{s_{i}}(x) \geq \varepsilon
$$

Although the CS model's heuristics and reasoning techniques deal with sensors' inaccuracy and characteristics of context attributes, they are inadequate to represent the uncertainty associated with real-life and human concepts which tend to be vague and uncertain. Changes that occur between situations are also indicators of vague and uncertain situations that need to be reflected in the situation inference results. A fuzzy approach has the strength to represent this level of uncertainty as well as minor and delta changes of context that can be important in certain applications.

The next section discusses the adaptation engine and describes how parameters of data stream mining algorithms are adjusted according to occurring situations and available resources.

\section{Adaptation Engine (AE)}

The AE (Adaptation Engine) is responsible for gradual tuning of data stream processing parameters according to the occurring situation/s and available resources in real time. AE provides three adaptation strategies including resourceaware, situation-aware and integrated strategies. The Controller is a subcomponent 
of $\mathrm{AE}$ that constantly monitors occurring situations that are inferred by FSI and availability of resources reported by RM. The Controller makes decisions on which strategy needs to be performed. AE and its components are shown in Fig. 3.

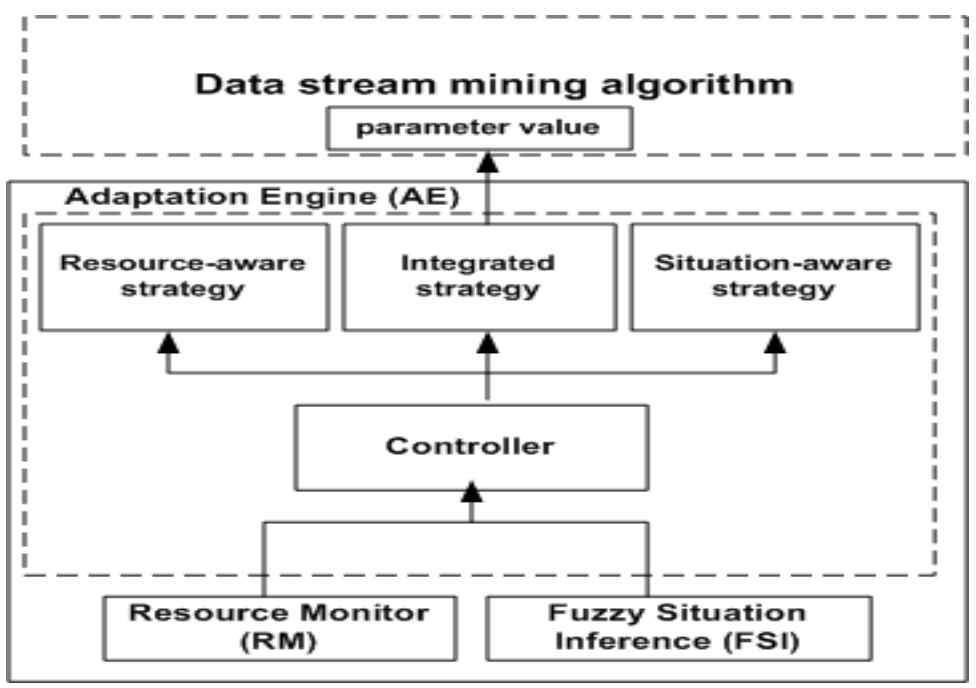

Fig. 3. Adaptation strategies of data stream mining algorithm.

Lightweight data stream mining techniques such LWC, LWCLass, LWF, RACluster [11-12] are adaptive to availability of resources via adjusting the algorithm parameters. These parameters control output, input and/or the process of the algorithm. The adaptation process is done through Algorithm Granularity (AG) approach. AG has three different variations. The first and most commonly used in our work is Algorithm Output Granularity $(A O G)$ [13]. AOG controls the algorithm output rate via changing the data stream mining algorithm parameters to encourage or discourage the creation of new output structures. Similarly Algorithm Input Granularity $(A I G)$ [12] and Algorithm Processing Granularity $(A P G)$ [12] control the input rate and consumption of processing power respectively.

LWC, LWClass and LWF algorithms are based on the AOG approach that controls the output of the data stream mining according to the available memory. We have inspired by the concept of AOG for developing our adaptation strategies.

To perform different strategies of adaptation, we need to pre-assign two thresholds for criticality of resources and occurring situations and specified by application designers. The thresholds of S_THRESHOLD_SAFE and R_THRESOLD_SAFE indicate the normal or safe level and S_THRESHOLD_CRITICAL and R_THRESOLD_CRITCIAL separate the medium and critical levels for situations and resource usage. For example if we have three situations of 'normal', 'pre-hypertension' and 'hypertension', we can assign the criticality values of $0.25,0.6,0.9$ to these situations respectively. The thresholds assigned to computational resources show the level of their criticality re- 
garding their usage. The higher the value is, the higher the usage is. Fig. 4 depicts the critical and safe thresholds of resources and situations.

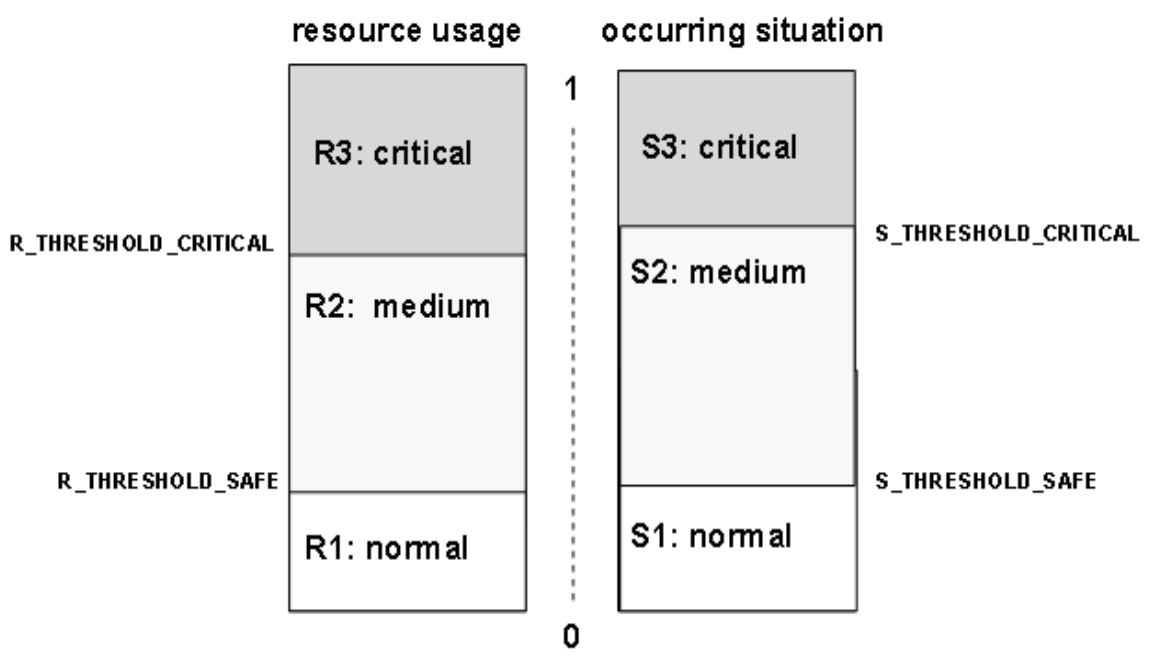

Fig. 4. Levels of criticality for resources and situations.

Based on the levels of criticality for situations and resources shown in Fig. 4., there are nine possible variations (cases) of adaptation that are considered by the Controller.

Table 2 present different variations of adaptation. Adaptation strategies are selected according to these nine cases.

Table 2. Adaptation cases.

\begin{tabular}{ll}
\hline Cases & Adaptation strategy \\
\hline $1-$ if R1 and S1 & Situation-aware strategy \\
2- if R1 and S2 & Situation-aware strategy \\
3- if R1 and S3 & Situation-aware strategy \\
4- if R2 and S1 & Resource-aware strategy \\
5- if R2 and S2 & Integrated strategy \\
6- if R2 and S3 & Integrated strategy \\
7- if R3 and S1 & Other strategies e.g. migration of data or mining process \\
8- if R3 and S2 & \\
$9-$ if R3 and S3 & \\
\hline
\end{tabular}


To formalize the adaptation strategy we use the notation in Table 3.

Table 3. Adaptation strategies symbols.

\begin{tabular}{ll}
\hline Symbol & Meaning \\
\hline $\boldsymbol{R} \boldsymbol{C}$ & Vector of resource criticality values $\left\{C\left(R_{1}\right), \ldots, C\left(R_{n}\right)\right\}$ \\
$C\left(R_{i}\right)$ & Criticality value of a resource $R_{i}$ (a value between 0-1) where \\
& $R_{i} \in\{$ memory, battery, $C P U\}$ \\
$\boldsymbol{I S}$ & Vector of occurring situations and their membership degree \\
& $\left(\left\{S_{1}, \mu_{1}\right\}, \ldots,\left\{S_{n}, \mu_{n}\right\}\right)$ \\
$S \boldsymbol{C}$ & Vector of situation criticality values $\left\{C\left(S_{1}\right), \ldots, C\left(S_{n}\right)\right\}$ \\
$C\left(S_{i}\right)$ & Criticality value of a situation $S_{i}($ a value between $0-1)$ \\
$S\left(\max \left(\mu_{i}\right)\right)$ & Function returning a situation with the highest membership degree \\
$\boldsymbol{H} S$ & The situation with the highest degree of membership \\
$C(H S)$ & Criticality value of HS \\
$P_{i}$ & The parameter to be adjusted \\
$P A_{i}$ & Adjusted value of the parameter $P_{i}$
\end{tabular}

The algorithm for selecting adaptation strategies uses the notation presented in Table 3 is shown in Fig. 5. This algorithm describes how each strategy is selected according to available resources and occurring situations.

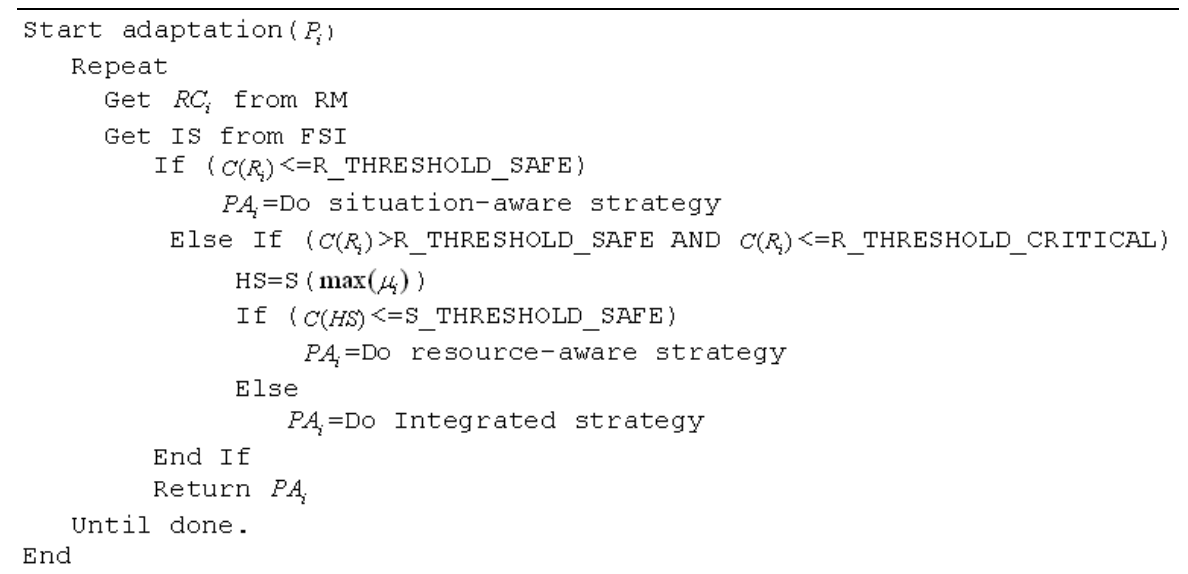

Fig. 5. The algorithm for adaptation of parameters 
When resources are critical (R3) it means that the mobile device can not continue the mining operations and the adaptation strategies that we provide are not adequate to address the issue. These cases require other strategies such as migration of the data or the process that are out of the scope of this project. Therefore the algorithm depicted in Fig. 5 does not deal with this issue.

The next subsection discusses the resource-aware adaptation strategy that factors in available resources in the adaptation phase.

\subsection{Resource-Aware Strategy}

Resource-aware adaptation strategy occurs when the situation is at normal level but resource availability is at medium level. This is because normal situations do not require frequent monitoring and the results of resource-aware adaptation do not contradict the requirements of normal situations. Resource-awareness is inspired by the AOG approach. One of the AOG-based clustering algorithms is called LightWeight Clustering (LWC) [11]. LWC considers a threshold distance measure for clustering of data. Increasing this threshold discourages forming of new clusters and in turn reduces resource consumption.

AOG is a three-stage, resource-aware distance-based mining data streams approach. The process of mining data streams using AOG starts with a mining phase. In this step, a value of threshold distance measure is determined. This threshold has the ability to control the output rate of the running mining algorithm. The second stage in AOG-mining approach is the adaptation phase. In this phase, the threshold value is adjusted to cope with the data rate of the incoming stream, available memory, and time constraints to fill the memory with generated knowledge (data mining output). The last stage in AOG approach is the knowledge integration phase. This stage represents the merging of generated results when the memory is full. This integration allows the continuity of the mining process on resource-constrained devices. Fig. 6 shows how AOG is applied to the LWC.

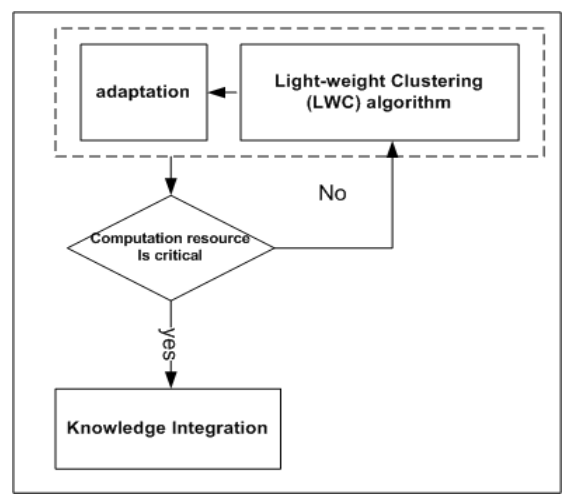

Fig. 6. Light-Weight Clustering algorithm based on AOG 
The details of how data stream mining algorithms are adjusted according to occurring situations are given in the next subsection.

\subsection{Situation-Aware Strategy}

Situation-aware adaptation in AE is performed when resources are available and at normal level. Situation-aware adaptation occurs based on occurring situations inferred by FSI. These results are multiple situations with different level of confidence. To provide a fine-grained adaptation and reflect the level of confidence of each situation in the adaptation phase, we compute weighted average of the data mining parameter value based on confidence values of situations and the prespecified value of the parameter for each situation. The situation-aware adaptation enables reflecting all the results of situation inference in the adaptation of parameter values and is represented as follows:

$$
\hat{p}_{j}=\sum_{i=1}^{n} \mu_{i} p_{j} / \sum_{i=1}^{n} \mu_{i}
$$

where $p_{j}$ represents the set value of a parameter for a pre-defined situation $S_{i}, \mu_{i}$ denotes the membership degree of situation $S_{i}$ where $1 \leq \mathrm{i} \leq \mathrm{n}$ and $\mathrm{n}$ represents the number of pre-defined situations, and $\hat{p}_{j}$ represents aggregated value of the parameter. Fig. 7 shows an example of situation-aware adaptation of LWC for three pre-defined situations of 'normal', 'hypertension' and 'hypertension' at the time t. To start the adaptation phase, the threshold parameter values are pre-set for each situation accordingly.

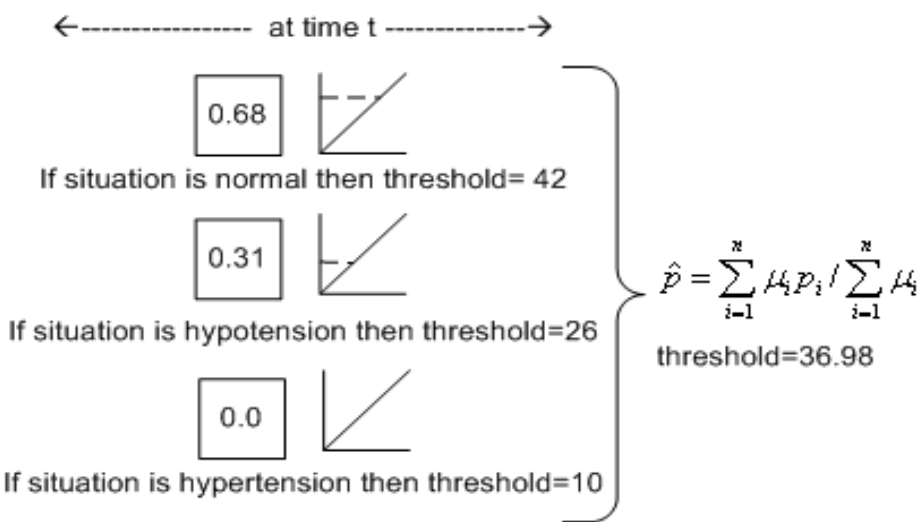

Fig. 7. An example of situation-aware adaptation strategy. 
Situation-aware adaptation itself results in cost-efficiency because when a situation is at normal level, the set value of parameter for a normal situation (e.g. a higher LWC threshold value) will be used and this reduces the use of resources.

The next subsection describes how integrated adaptation strategy combines resource-aware and situation-aware strategies and deals with the trade-off.

\subsection{Integrated Strategy}

When criticality value of resources is at medium level and situation criticality value is at medium or critical level (i.e. R2S2 or R2S3), it is imperative to consider both resource availability and occurring situations in the adaptation phase.

When the adaptation cases R2S2 or R2S3 occur, resource-aware and situationaware adaptation strategies compute different values for parameters. This is because resource-aware strategy reduces resource consumption to deal with resource criticality (i.e. at medium level) and situation-aware strategy intends to increase accuracy of mining results by increasing output that is typically required in critical situations. This causes a trade-off between the adjusted values of parameters.

As an example, consider the criticality value of memory is 0.62 and the criticality value of occurring situation (i.e. with highest level of confidence) is 0.9 . To adjust the LWC's threshold parameter, the resource-aware strategy computes a higher threshold value (e.g. 32) to produce fewer results and conserve the memory but the situation-aware strategy computes a lower value (e.g. 12) to increase the output. The integrated strategy addresses this issue by computing the average value of parameter based on the results of the two strategies (i.e. $\hat{p}_{R}$ and $\hat{p}_{S}$ ) and criticality values as follows:

$$
\hat{p}_{I}=\frac{\left(\hat{p}_{R} \cdot \text { criticality }_{R}\right)+\left(\hat{p}_{S} \cdot \text { criticality }_{S}\right)}{\text { criticality }_{R}+\text { criticality }_{S}}
$$

Fig. 8 shows how this strategy can be used to deal with the above-described.

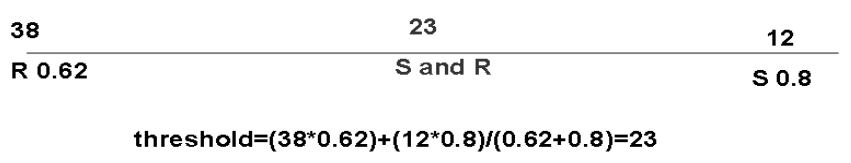

Fig. 8. An example of using resource-aware strategy

To validate SAAP we have performed evaluation of the reasoning technique of FSI and situation-aware adaptation. The next section describes these evaluations. 


\section{Evaluation}

For evaluation of SAAP, we have performed two evaluations. First evaluation is a comparative evaluation of FSI, CS and Dempster-Shafer to validate the FSI technique and highlight its benefits in dealing with uncertainty. Second evaluation focuses on adaptation of threshold parameter according to occurring situations.

\subsection{FSI Evaluation}

To evaluate the FSI model, we have compared the FSI situation reasoning technique to the CS and Dempster-Shafer (hereafter DS) reasoning approaches. The purpose of this evaluation is first to validate the FSI model against a well-known reasoning technique such as DS and a context model developed for pervasive computing environments such as the CS model.

The second objective of the evaluation is to highlight the benefits of the FSI to deal with uncertain context and situations. In this evaluation, we consider three situations of 'hypotension', 'normal' and 'hypertension' that are defined using context attributes of systolic (SBP) and diastolic blood pressure (DBP) with the scale of $40-170$ and $20-150 \mathrm{~mm} \mathrm{Hg}$ respectively and heart rate (HR) with the range of 20-150 bpm.

Table 4 depicts modeling of the three situations in the CS model including the weights of attributes and their corresponding regions of values. Unlike FSI, the CS model uses crisp boundary for regions. To provide a similar and balanced range of data for evaluation of these approaches, the boundaries of regions are selected in a way that they match the values of fuzzy sets with membership degree of 0.5 .

Table 4. Situation definitions in the CS model.

\begin{tabular}{llll}
\hline Situation & Context attribute & Region of values & Weight \\
\hline Hypotension & $1=$ SBP & $\leq 85$ & 0.4 \\
& $2=$ DBP & $\leq 60$ & 0.4 \\
$3=$ HR & $\leq 45$ & 0.2 \\
Normal & $1=$ SBP & $>85$ and $\leq 135$ & 0.4 \\
& $2=$ DBP & $>60$ and $\leq 110$ & 0.4 \\
Hypertension & 1=SBP & $>45$ and $\leq 85$ & 0.2 \\
& $2=$ DBP & $>135$ & 0.4 \\
$3=$ HR & $>110$ & 0.4 \\
\hline
\end{tabular}

Although FSI can represent a situation with multiple rules and each condition can be joined by the OR operator, we use one rule to define a situation and do not include the OR operator so that both models can be closely compared. The model- 
ing of the situations in FSI is presented in Table 5. Weights of conditions for the FSI rules conform to the weights specified for the context attributes in CS.

Table 5. Situation definitions in the FSI model.

\begin{tabular}{llll}
\hline Situation & Linguistic Variable & Terms & Fuzzy set \\
\hline represented below via FSI rules & $1=$ SBP & low, normal, high & trapezoidal member- \\
& $2=\mathrm{DBP}$ & low, normal, high & ship functions used \\
& $3=\mathrm{HR}$ & slow, normal, fast &
\end{tabular}

Rule1: if SBP is low and DBP is low and HR is low then situation is hypotension

Rule2: if SBP is normal and DBP is normal and HR is normal then situation is normal

Rule3: if SBP is high and DBP is high and HR is high then situation is hypertension

To model situations of Hypotension (L), Normal (N) and Hypertension (H) with DS, we first define propositions and events. Since all three situations are incompatible we include a proposition of Unknown (U) that would consist of three situations. Then we identify the events and mass values that reflect the association of an event with the occurrences of each proposition. Mass values are assigned in a way that they reflect to what degree each event indicates a situation. Table 6 depicts the events and mass values using the DS technique.

Table 6. Definitions of events and mass values.

\begin{tabular}{|c|c|c|c|c|c|}
\hline Event & $\begin{array}{l}\text { Mass values } \\
\text { for Normal }\end{array}$ & $\begin{array}{l}\text { Mass values } \\
\text { for Hypotension }\end{array}$ & $\begin{array}{l}\text { Mass values } \\
\text { For Hypertension }\end{array}$ & $\begin{array}{l}\text { Mass values } \\
\text { for unknown }\end{array}$ & $\begin{array}{l}\text { Total } \\
\text { mass }\end{array}$ \\
\hline SBPLow (40-85) & 0 & 0.7 & 0 & 0.3 & 1 \\
\hline SBPMed(86-135) & 0.7 & 0 & 0 & 0.3 & 1 \\
\hline SBPHigh(136-180) & 0 & 0 & 0.7 & 0.3 & 1 \\
\hline DBPLow(20-60) & 0 & 0.7 & 0 & 0.3 & 1 \\
\hline DBPMed(61-110) & 0.7 & 0 & 0 & 0.3 & 1 \\
\hline DBPHigh(110-130) & 0 & 0 & 0.7 & 0.3 & 1 \\
\hline HRSlow(20-45) & 0.2 & 0.4 & 0 & 0.4 & 1 \\
\hline HRMed(46-85) & 0.4 & 0.2 & 0.2 & 0.2 & 1 \\
\hline HRFast(86-130) & 0.2 & 0 & 0.4 & 0.4 & 1 \\
\hline
\end{tabular}

To apply the DS algorithm for situation reasoning, we use the Dempster's rule of combination. If $\mathrm{R}$ represents a situation, considering all existing propositions, the intersection of some of these propositions denoted as $\mathrm{P}$ and $\mathrm{Q}$ results in the proposition $\mathrm{R}$ (i.e. $P \cap Q=R$ ) and the intersection of other combinations of propositions results in an empty set. The normalized version of the combination rule of DS is as follows. 


$$
m(R)=\frac{\sum_{P \cap Q=R} m_{i}(P) \cdot m_{j}(Q)}{1-\sum_{P \cap Q=\phi} m_{i}(P) \cdot m_{j}(Q)}
$$

where $m(R)$ denotes the mass value computed for a proposition $R$ given the evidences $\mathrm{i}$ and $\mathrm{j}$.

Since we have based our situations on three context attributes, we define three mass functions of $m_{1}, m_{2}$ and $m_{3}$ corresponding to each context attribute. Then we apply DS combination over all propositions and available evidence. For example, if we have the context values of 82 for SBP, 52 for DBP and 58 for HR, we combine evidence for the occurrence of hypotension (L) as follows.

$$
\begin{aligned}
& m_{12}(L)=\frac{\sum_{Q \cap R=L} m_{1}(A) \cdot m_{2}(B)}{1-\sum_{Q \cap R=\phi} m_{1}(A) \cdot m_{2}(B)}=\frac{m_{1}(L) \cdot m_{2}(L)+m_{1}(L) \cdot m_{2}(U)+m_{1}(U) \cdot m_{2}(L)}{1-m_{1}(L) \cdot m_{2}(H)-m_{1}(H) \cdot m_{2}(L)-m_{1}(L) \cdot m_{2}(N)-m_{1}(N) \cdot m_{2}(L)}= \\
& \frac{0.7 \cdot 0.7+0.7 \cdot 0.3+0.3 \cdot 0.7}{1-0.7 \cdot 0-0 \cdot 0.7-0.7 \cdot 0-0 \cdot 0.7} \approx 0.91
\end{aligned}
$$$$
m_{12}(H)=\frac{\sum_{Q \cap R=H} m_{1}(A) \cdot m_{2}(B)}{1-\sum_{Q \cap R=\phi} m_{1}(A) \cdot m_{2}(B)}=\frac{m_{1}(H) \cdot m_{2}(H)+m_{1}(H) \cdot m_{2}(U)+m_{1}(U) \cdot m_{2}(H)}{1-m_{1}(H) \cdot m_{2}(L)-m_{1}(L) \cdot m_{2}(H)-m_{1}(H) \cdot m_{2}(N)-m_{1}(N) \cdot m(H)}=
$$$$
\frac{0 \cdot 0+0 \cdot 0.3+0.3 \cdot 0}{1-0 \cdot 0.7-0.7 \cdot 0-0 \cdot 0-0 \cdot 0}=0
$$$$
m_{12}(N)=\frac{\sum_{Q \cap R=N} m_{1}(A) \cdot m_{2}(B)}{1-\sum_{Q \cap R=\phi} m_{1}(A) \cdot m_{2}(B)}=\frac{m_{1}(N) \cdot m_{2}(N)+m_{1}(N) \cdot m_{2}(U)+m_{1}(U) \cdot m_{2}(N)}{1-m_{1}(N) \cdot m_{2}(L)-m_{1}(L) \cdot m_{2}(N)-m_{1}(N) \cdot m_{2}(H)-m_{1}(H) \cdot m(N)}=
$$$$
0 \cdot 0+0 \cdot 0.3+0.3 \cdot 0
$$$$
\frac{0 \cdot 0+0 \cdot 0.3+0.3 \cdot 0}{1-0 \cdot 0.7-0.7 \cdot 0-0 \cdot 0-0 \cdot 0}=0
$$

$m_{12}(U)=\frac{\sum_{Q \cap R=U} m_{1}(A) \cdot m_{2}(B)}{1-\sum_{Q \cap R=\phi} m_{1}(A) \cdot m_{2}(B)}=$

$\frac{m_{1}(U) \cdot m_{2}(U)}{1-m_{1}(H) \cdot m_{2}(L)-m_{1}(L) \cdot m_{2}(H)-m_{1}(N) \cdot m_{2}(H)-m_{1}(H) \cdot m(N)-m_{1}(N) \cdot m_{2}(L)-m_{1}(L) \cdot m_{2}(N)}=$

$\frac{0.3 \cdot 0.3}{1-0 \cdot 0.7-0.7 \cdot 0-0 \cdot 0-0 \cdot 0-0.0 .7-0.7 .0} \approx 0.09$

$m_{123}(L)=\frac{\sum_{Q \cap R=L} m_{1}(A) \cdot m_{2}(B)}{1-\sum_{Q \cap R=\phi} m_{1}(A) \cdot m_{2}(B)}=\frac{m_{12}(L) \cdot m_{3}(L)+m_{12}(L) \cdot m_{3}(U)+m_{3}(U) \cdot m_{12}(L)}{1-m_{12}(L) \cdot m_{3}(H)-m_{12}(H) \cdot m_{3}(L)-m_{12}(L) \cdot m_{3}(N)-m_{12}(N) \cdot m_{3}(L)}=$

$\frac{0.91 \cdot 0.2+0.91 \cdot 0.2+0.09 \cdot 0.2}{1-0.91 \cdot 0.2-0 \cdot 0.2-0.91 \cdot 0.4-0 \cdot 0.2}=\frac{0.382}{0.454} \approx 0.841$

The same DS reasoning computation presented above is used in our evaluation. Although, the DS theory has the strength of representing unknown or uncertainty, 
determination of mass values for propositions can be a difficult task, particularly that they can have impact on the other situations. For evaluation of CS and FSI, we use the equations 1 and 2 (discussed in subsection 4.2). These techniques do not include the sensor's inaccuracy and could be compared to the DS method more accurately.

The dataset used for evaluation is generated continuously (data rate is 30 records/minute) in ascending order to model the changes of situations. For this set of experiments, we have used our data synthesizer to represent the seven different situations based on the description in Table 6. This categorization shows the data scales that contribute to the occurrence of each pre-defined situation as well as the uncertain situations. Table 7 depicts a snapshot of 131 context states that is used along with their scales.

Table 7. The dataset used for the evaluation

\begin{tabular}{ll}
\hline Context attribute scales & Corresponding DS events \\
\hline SBP:40-65, DBP: 20-45, HR: 20-45 & SBPLow, DBPLow, HRSlow \\
SBP:66-80, DBP: 46-60, HR: 46-60 & SBPLow, DBPLow, HRMed \\
SBP:81-85, DBP: 61-65, HR: 61-65 & SBPLow, DBPMed, HRMed \\
SBP:86-105, DBP: 66-85, HR: 66-85 & SBPMed, DBPMed, HRMed \\
SBP:106-130, DBP: 86-110, HR: 86-110 & SBPMed, DBPMed, HRHigh \\
SBP:131-135, DBP: 111-115, HR: 111-115 & SBPLow, DBPHigh, HRHigh \\
SBP:136-170, DBP: 116-150, HR: 116-150 & SBPHigh, DBPHigh, HRHigh \\
\hline
\end{tabular}

The results of the comparative evaluation of CS, DS and FSI for situations of 'hypotension', 'normal' and 'hypertension' in Fig. 9 show that three approaches of CS, DS and FSI have a relatively similar trend according to context changes. When the data corresponds to a pre-defined situation the results of three approaches almost overlap. This overlapping is more noticeable with the CS and FSI models as they are based on similar heuristics. However, when changes of data indicate the occurrence of an unknown and uncertain situation, differences of reasoning results between CS, DS and FSI are more apparent.

Compared to FSI, the results of situation reasoning by the CS and DS methods show sudden rise and fall with sharp edges when situations change which do not match the real-life situations. This is because the DS and CS approaches do not deal with delta changes of the values and are not able to reflect the gradual evolution of one situation to another situation.

When the value of context attributes decreases or increases, its membership degree also increases and decreases accordingly and gradually. This enables FSI to provide more accurate situation reasoning results in terms of reflecting very minor changes of context. 

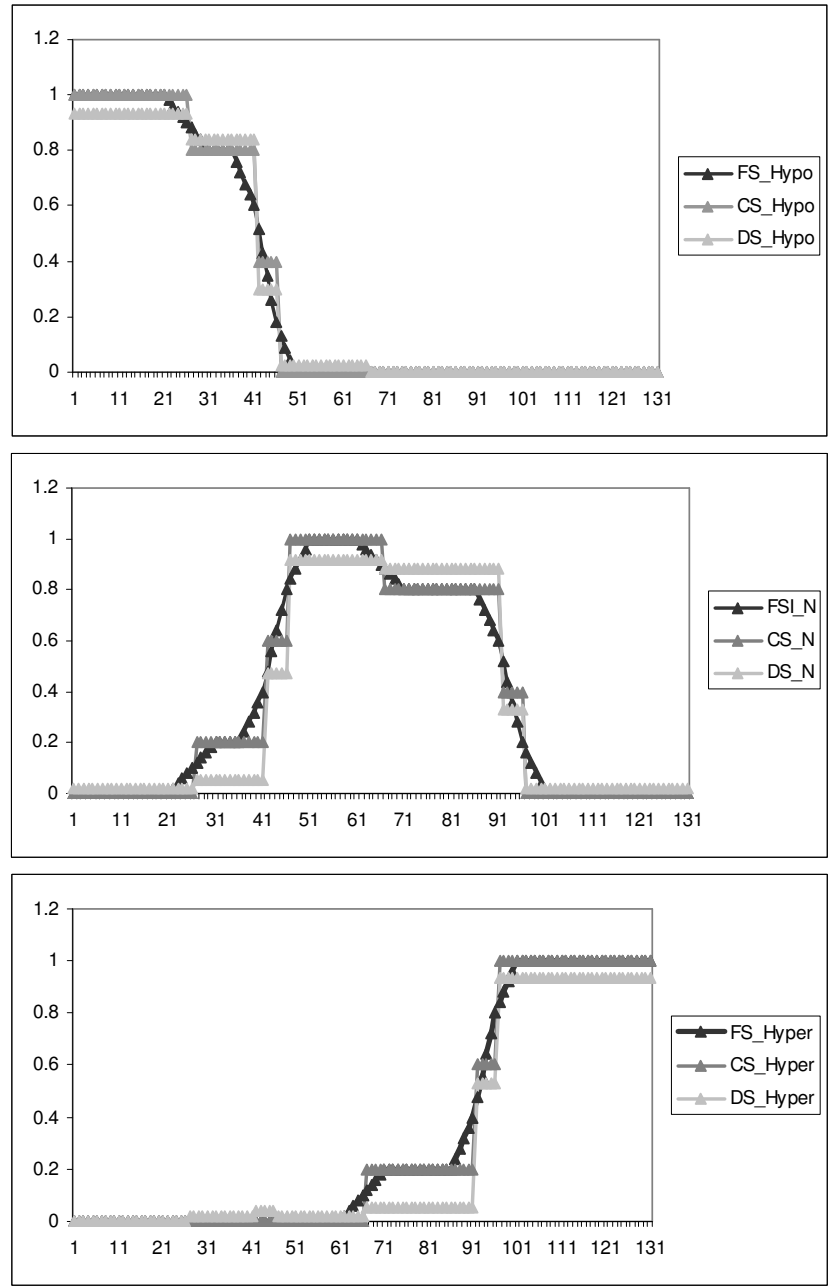

Fig. 9. The results of the comparative evaluation

The evaluation validates the accuracy of the FSI model for situation modeling and reasoning and it also shows that FSI is able to reflect very minor changes of context in situation inference and represent changes in a more gradual and smooth manner. The evaluation shows that the FSI model is more appropriate approach for representation of human concepts and for reasoning about the real-world situations that are defined by continuous values. Health-related situations are examples of these types of scenarios where FSI can prove to be more fitting approach compared to the DS and CS reasoning approaches. 


\subsection{Evaluation of Situation-aware Adaptation}

Data mining algorithm that we have used for evaluation is LightWeight Clustering (LWC) [9]. The threshold distance parameter of the LWC algorithm determines the distance between the center of a cluster and a new incoming data record. In our evaluation, we have set the threshold value for the situation of 'normal' to 42 , 'pre-hypotension' to 36 , 'hypotension' to 26 , 'pre-hypertension' to 18 and 'hypertension' to 10 . For critical situations the threshold needs to be decreased and for normal situations the threshold needs to be increased. This is because these values are acceptable given a variation of 12 (i.e. 42 divided by 3 ) for any of the context attributes of SBP, DBP and HR has no significant impact on a healthy individual while a variation of 3 for 'hypertension' can be significant.

To analyze the results of situation reasoning and adaptation for all the situations, we have simulated a 5-day scenario for a patient that experiences fluctuations of blood pressure. The scenario is described in Table 8 . The dataset used for the evaluation is drawn from uniform distribution with different ranges for each context attribute (i.e. SBP, DBP and HR). The date is generated at a rate of 30 records/minute. The rate was chosen according to the application needs. This rate could be customized to meet the different requirements of various applications.

Table 8. A 5-day scenario.

\begin{tabular}{ll}
\hline Day & \multicolumn{1}{c}{ Patient's health state } \\
\hline 1 & Vital signs are normal and RT is mild \\
2 & Morning: RT is gradually rising and SBP, DBP and HR are decreasing \\
& Afternoon: SBP, DBP and HR are very low ( necessary medical treatment provided) \\
& Evening: SBP, DBP and HR are gradually increasing and RT is dropping \\
3 & Vital signs are normal and RT is mild \\
4 & Morning: SBP, DBP and HR are increasing \\
& Afternoon: SBP, DBP and HR are very high ( necessary medical treatment provided) \\
5 & Evening: SBP, DBP and HR are gradually decreasing \\
\hline
\end{tabular}

Fig. 10 illustrates the results of this evaluation. The top graph shows changes of context attribute values for each day and the bottom graph shows the corresponding fuzzy situations. As the values of SBP, DBP and HR decrease (i.e. day 2), the membership degrees of 'hypotension' and 'pre-hypotension' situations increase and as these values increase (i.e. day 4), the membership degrees of 'hypertension' and pre-hypertension' increase. Furthermore, the results of situation inference for day 2 and 4 also reflect vague situations when the patient is recovering or moving towards a situation (e.g. 'more or less normal' and 'slightly pre-hypertension'). 


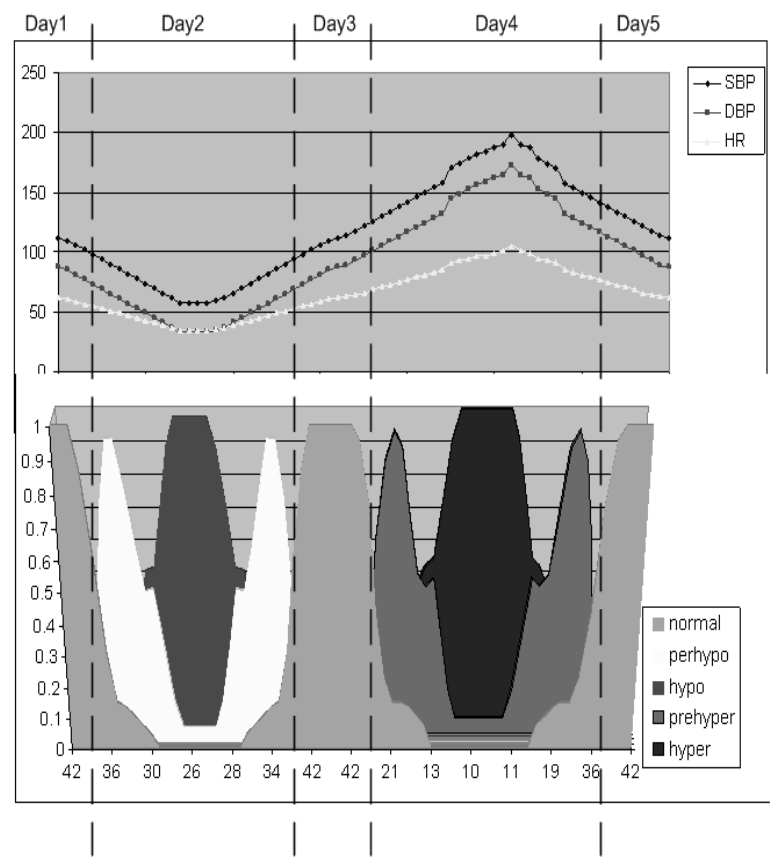

Fig. 10. The results of situation-aware adaptation.

The bottom graph in Fig. 10 shows how the results of situation inference are used for gradual adjusting of the distance threshold value. The value of the threshold is automatically adjusted according to the fuzziness (i.e. membership degree) of each situation in run-time. Decreasing the threshold value increases the number and accuracy of the output (clusters) that is required for closer monitoring of more critical situations such as hypertension. Alternatively, increasing the threshold value for normal situations decreases the mining output and also provides cost-efficiency of resources.

\section{Conclusion}

Ubiquitous applications such as healthcare monitoring applications need to analyze and process data streams that are generated at very high rates in real-time. Therefore it is of great importance for data stream mining techniques to be equipped with adapting strategies to promote the continuity and consistency of the running application. Current ubiquitous data stream mining approaches have limited levels of adaptations (mainly focusing on battery or memory). To enhance adaptation of data stream mining algorithms, there is a need to consider the con- 
textual/situational information in the adaptation phase. Integrating data stream processing with situation-awareness provides intelligent and cost-efficient analysis of data and enables continuity and consistency of mining operations.

In this chapter we proposed and validated a general approach for situationaware adaptive processing (SAAP) of data streams that incorporates situationawareness into data stream processing using fuzzy logic. The fuzzy situation inference model allows modeling and reasoning about real-world. The SAAP architecture enables real-time analysis of data emanating from multiple sensors onboard mobile devices while factoring in contextual/situational information and resource availability. This approach significantly enhances a range of ubiquitous applications that perform data stream mining on mobile devices.

In future, we intend to investigate other strategies of adaptation that can deal with criticality of resources and situations such as migration of data or mining process to neighboring devices. We also aim to explore and model relationships between situations, and extend FSI with learning capabilities so the system can predict future situations.

\section{References}

[1] Gaber MM, Krishnaswamy S, Zaslavsky A (2005) Resource-Aware Mining of Data Streams. Journal of Universal Computer Science. 11(8): 1440—1453

[2] Gaber MM, Zaslavsky A, Krishnaswamy S (2004) A Cost-Efficient Model for Ubiquitous Data Stream Mining, Proceedings of the Tenth International Conference on Information Processing and Management of Uncertainty in Knowledge-Based Systems, Perugia Italy

[3] Kargupta H, Bhargava R, Liu K, Powers M, Blair P, Bushra S, Dull J, Sarkar K, Klein M, Vasa M, Handy D (2004) VEDAS: A Mobile and Distributed Data Stream Mining System for Real-Time Vehicle Monitoring. In Proceedings of the SIAM International Data Mining Conference, SDM'04

[4] Galan M, Liu H, Torkkola K (2005) Intelligent Instance Selection of Data Streams for Smart Sensor Applications. SPIE Defense and Security Symposium, Intelligent Computing: Theory and Applications III: 108-119

[5] Padovitz A, Zaslavsky A, Loke S (2006) A Unifying Model for Representing and Reasoning About Context under Uncertainty, 11th International Conference on Information Processing and Management of Uncertainty in Knowledge-Based Systems (IPMU), Paris, France

[6] Anagnostopoulos CB, Ntarladimas Y, Hadjiefthymiades S (2007) Situational Computing: An Innovative Architecture with Imprecise Reasoning, the Journal of Systems and Software 80: 1993-2014

[7] Jang JR, Sun Ch, Mizutani E (1997) Neuro-Fuzzy and Soft Computing: A Computational Approach to Learning and Machine Intelligence. Prentice-Hall: Upper Saddle River, NJ. 
[8] Zadeh L (1975) The Concept of a Linguistic Variable and Its Application to Approximate Reasoning. Information Systems: 199-249.

[9] Zimmermann H (1996) Fuzzy Set Theory - and Its Applications. Kluwer Academic Publishers, Norwell, Massachusetts.

[10] Bruce G, Buchanan BG, Shortliffe ED (1984) Rule-based expert systems : the MYCIN experiments of the Stanford Heuristic Programming Project. Reading, Mass: Addison-Wesley.

[11] Gaber MM, Krishnaswamy S, Zaslavsky A (2005) On-board Mining of Data Streams in Sensor Networks", A Book Chapter in Advanced Methods of Knowledge Discovery from Complex Data, (Eds.) S. Badhyopadhyay, U. Maulik, L. Holder and D. Cook, Springer Verlag.

[12] Phung N, Gaber MM, Roehm U (2007) Resource-aware Distributed Online Data Mining for Wireless Sensor Networks, Proceedings of the International Workshop on Knowledge Discovery from Ubiquitous Data Streams (IWKDUDS07), in conjunction with ECML and PKDD 2007, Warsaw, Poland.

[13] Gaber MM, Krishnaswamy S, Zaslavsky A (2003) Adaptive Mining Techniques for Data Streams Using Algorithm Output Granularity, The Australasian Data Mining Workshop (AusDM 2003), Held in conjunction with the 2003 Congress on Evolutionary Computation (CEC 2003), Canberra, Australia, Springer Verlag, Lecture Notes in Computer Science (LNCS) 